\title{
Análise da versão espanhola do Sport Satisfaction Instrument (SSI) adaptado à Educação Física
}

\author{
Antonio Granero-Gallegos ${ }^{3}$ \\ Antonio Baena Extremera ${ }^{1}$ \\ Humberto Luís de Deus Inácio ${ }^{2}$ \\ Clara Bracho-Amador ${ }^{3}$ \\ Francisco Javier Pérez-Quero ${ }^{3}$ \\ Facultad de Ciencias del Deporte, Universidad de Murcia, España \\ ${ }^{2}$ Faculdade de Educação Física. Universidade Federal de Goiás, Goiânia, GO, Brasil \\ ${ }^{3}$ Centro del Profesorado Almería 03, Consejería de Educación, Junta de Andalucía, España
}

\begin{abstract}
Resumo: O objetivo deste estudo foi analisar as propriedades psicométricas do Sport Satisfation Instrument (SSI) adaptado para a Educação Física (EF) por meio de uma análise fatorial exploratória da estrutura bidimensional do instrumento em uma amostra espanhola. Com isso, buscou-se determinar, de maneira preliminar, se o SSI constitui um instrumento válido e fiável para ser utilizado em futuras pesquisas. $\mathrm{O}$ instrumento foi elaborado em um modelo teórico de dois fatores: Satisfação/Diversão e Tédio. A amostra constituiu-se de um total de 224 alunos de secundária entre 12 e 19 anos. A versão [espanhola] do instrumento adaptado para a EF demonstrou níveis aceitáveis de consistência interna.
\end{abstract}

Palavras-chave: Educação Física. Satisfação. Tédio. Propriedades psicométricas. SSI.

\section{Analysis of the Spanish version of the Sport Satisfaction Instrument (SSI) adapted to Physical Education}

\begin{abstract}
The objective of this study was to analyze the psychometric properties of Sport Satisfaction Instrument (SSI) adapted physical education (PE) using exploratory factor analysis of the dimensional structure of the instrument in a Spanish sample. It was intended to determine, on a preliminary basis, whether it constitutes a valid and reliable for use in future research. Was administered to a total of 224 high school students 12 to 19 years. This analysis supports the hypothesized theoretical model of two factors (satisfaction / fun and boredom). The Spanish version of the instrument for PE showed acceptable levels of internal consistency.
\end{abstract}

Keywords: Physical Education. Satisfaction. Boredom. Psychometric properties, SSI.

\section{Introdução}

A satisfação com a escola e com o esporte tem sido investigada por vários autores, há décadas, como um fator determinante da motivação dos alunos em seu desempenho acadêmico e desportivo e até mesmo na adesão ao esporte (BALAGUER, 2000. STUCKY-ROPP; DILORENZO, 1993).

A satisfação é definida como o grau de diversão ou tédio experimentado por pessoas em certa atividade (Castillo et al., 2002. DUDA e NICHOLLS, 1992) e pode levar a um aumento daquelas atividades que são divertidas (DUDA, 1993) ou a exclusão das que são entediantes (ROBERTS, 1984).

Sobre estas variáveis motivacionais, os primeiros estudos foram realizados por Nicholls et al. (1989) e Nicholls et al. (1985), os quais desenvolveram a Intrinsic Satisfaction Classroom Scale (ISC). Com esta escala, desenvolveram um trabalho com 587 alunos de várias escolas, medindo, através dela, o grau de satisfação e o interesse intrínseco dos alunos em relação à escola.

A ISC é composta por 8 itens divididos em duas escalas de medição de Satisfação/Diversão (5 itens) e Tédio ( 3 itens) na prática de atividades escolares/acadêmicas. Anos mais tarde, Duda e Nicholls (1992) realizaram uma adaptação do ISC para o esporte, dando origem ao Instrumento de Satisfação do Esporte (SSI). Com ambas as escalas realizaram um estudo com 207 adolescentes, com idade média $(M$ ) de 15,1 anos. $A$ análise fatorial revelou dois fatores para ambas as escalas, havendo três itens para 0 fator de Tédio, com um alfa de Cronbach ( $\alpha$ ) de 0,71 , e cinco itens para o fator de Satisfação/Diversão ( $\alpha$ $=0,82$.)

Embora estas escalas possibilitem obter informações de grande relevância no campo da 
motivação, tanto para a escola como para os esportes, há poucos estudos desenvolvidos no campo dos esportes e, muito menos, em relação à escola. Na Espanha, Balaguer et al. (1997) realizaram um trabalho com a escala SSI (Satisfação Intrínseca no Sport), desenvolvendo uma análise fatorial confirmatória da escala entre estudantes espanhóis, tornando-se a versão de referência no país. Nesta versão, os coeficientes alfa de Cronbach variaram de Satisfação/Diversão $(\alpha=0,82)$, e Tédio $(\alpha=0,71)$, confirmando o trabalho de Duda e Nicholls (1992) e Nicholls et al. $(1985,1989)$.

A proposta de Balaquer et al. (1997) foi também utilizada em pesquisas por Castillo et al. (2001) e Duda, (2002), com 967 alunos com idades entre 11 e 16 anos, na cidade de Valência. Esses autores também comprovam a existência das duas dimensões: Satisfação/Diversão $(\alpha=$ $0,80)$ e Tédio $(\alpha=0,76)$, com 5 e 2 itens, respectivamente. A análise de fiabilidade revelou que a supressão do item 2 da escala de Tédio, aumentou o coeficiente alfa de Cronbach da escala de um valor inicial de 0,62 para 0,76 . Realizaram ainda uma análise fatorial confirmatória do instrumento, mostrando uma aderência adequada dos índices de ajuste. Outros estudos, como Castillo et al. (2004), em uma amostra de 967 estudantes de 11 a 16 anos, obtiveram para Satisfação/Diversão $\alpha=0,82$ para meninos e $\alpha=0,87$ para meninas; e o Tédio, $\alpha=$ 0,74 para meninos e $\alpha=0,78$ para meninas.

No que diz respeito à utilização do ISC na Espanha, os trabalhos de Nicholls et al. (1985, 1989), Duda e Nicholls (1992), Balaguer et al. (1997) e Castillo et al. (2002), forneceram evidências empíricas da validade de construto desta escala. O modelo testado indicou a hipótese da existência de duas variáveis latentes dependentes: Satisfação/Diversão (5 itens) e Tédio ( 3 itens); ainda que - no primeiro, tivéssemos $\alpha=0,80$, no segundo fator se eliminou o item número 2 para aumentar a consistência interna até 0,76 . Assim, os resultados da análise confirmatória mostraram alguns índices de aderência.

Importa lembrar que a motivação nas/para as aulas de EF é tema de debate há muito tempo. Desta problemática derivam-se outras tais como: o professor de EF é mais um amigo que um professor, a aula de EF é um tempo de recuperação 'mental' em função das aulas das 'disciplinas teóricas', a aula de EF é um passatempo etc. E ainda, em tempos atuais, nos quais a prática do esporte/exercício físico vem sendo paulatinamente substituída por horas diárias à frente de um computador ou televisor, a EF passa a ser percebida como um momento de tédio, estafante, indesejado.

Assim, seja pela atração do passatempo associada a um período de 'descanso mental', seja pela aversão provocada por uma tendência crescente ao sedentarismo, urge que se compreenda com claridade 0 que sentem, percebem e asseveram alunos e alunas de EF sobre o(s) momentos(s) da aula.

É nesta direção que, desde os trabalhos até aqui apresentados, foi elaborada uma adaptação da escala SSI (BALAGUER et al., 1997) para a Educação Física, com o objetivo de estudar as propriedades psicométricas da mesma [escala]. Assim, este estudo realizou uma avaliação preliminar do SSI adaptado à EF, por meio de uma análise fatorial exploratória da estrutura dimensional do instrumento e uma análise de confiabilidade da escala, procurando indicar um instrumento fiável e consistente para avaliar o que pensam/sentem os alunos e alunas de EF.

\section{Método}

\section{Participantes}

Este primeiro estudo incluiu um total de 224 alunos $(110$ homens $=49,1 \%, 114$ mulheres $=$ $50,9 \%$ ) de duas escolas secundárias ${ }^{1}$ da província de Almeria. Este é um ' $n$ ' bem acima do número mínimo de indivíduos necessário para um estudo piloto de uma escala (OSTERLIND, 1989). Além disso, o fato de serem duas escolas, e não apenas uma, confere validez aos dados coletados, uma vez que oriundos de realidades (fontes) distintas. A faixa etária foi de 12 a 19 anos $(M=14,68, S D=1,27)$, com uma média de idade dos meninos 14,90 ( $D P=1,23)$, e das meninas $14,47(S D=1,28)$. A distribuição dos níveis de ensino foi como segue: $79(35,3 \%)$ do $2^{\circ}$. ESO; 45 (20,1\%), do 3‥ ESO; $44(19,6 \%)$, do $4 \%$. ESO e, finalmente, $56(25 \%)$, do $1^{\circ}$. bachillerato $^{2}$.

\footnotetext{
1 O nível de "escola secundária" espanhola apresenta a seguinte equivalência com o sistema de ensino brasileiro: $2^{\circ}$ $E S O=7^{\circ}$ ano, $3^{\circ} E S O=8^{\circ}$ ano, e $4^{\circ} E S O=9^{\circ}$ ano $-d o$ Ensino Fundamental.

$201^{\circ}$ "bachillerato" corresponde aos $1^{\circ}$ e $2^{\circ}$ anos do Ensino Médio.
} 


\section{Instrumento}

O instrumento de coleta de dados foi o Sport Satisfaction Instrument (SSI) adaptado (BALAGUER et al. 1997. DUDA e NICHOLLS, 1992). É composto de 8 itens para medir a satisfação intrínseca em uma atividade esportiva, com duas subescalas que medem a Satisfação/Diversão (5 itens) e o Tédio (3 itens). Nas instruções se pediu aos alunos para indicar 0 grau de concordância com os itens que refletem critérios de diversão ou tédio, coletando-se respostas em uma escala de itens poliatômicos de 5 pontos que variam desde discordo totalmente (1) a muito de acordo (5). A consistência interna da subescala de Satisfação/Diversão $=\alpha$ foi 0,92 e a de Tédio $\alpha=$ 0,78 .

\section{Processo de adaptação}

Como dito anteriormente, foi elaborada uma adaptação da versão espanhola do SSI por Balaguer et al. (1997), quando estudou o esporte; este mesmo instrumento adaptado foi utilizado em nossa investigação. A avaliação qualitativa (validade de conteúdo) foi feita pelo julgamento de quatro especialistas (OSTERLIND, 1989): dois em construção de escalas e dois conhecedores do construto a avaliar. Eles receberam uma tabela de especificações dos itens (CALABUIG; CRESPO, 2009. SPAAN, 2006), pela qual se instaurou a definição semântica do construto a ser avaliado e a de seu componente; também Ihes foi mostrada a lista de itens após a adaptação dos originais.

Eles deveriam emitir um juízo sobre sua relevância e sua compreensão numa escala desde 1 (discordo plenamente) a 4 (concordo plenamente). Além disso, havia uma seção para anotações e comentários gerais sobre cada um dos itens, pode também apresentar comentários gerais sobre cada item, se considerassem adequado. Os itens cuja média foi $<3$, tanto na relevância como na compreensão, foram revistos (NUVIALA et al., 2008). Se o item não fosse bem avaliado por pelo menos 3 dos 4 peritos nas dimensões já conhecidas (Satisfação/Diversão e Tédio), era novamente analisado, em busca de problemas potenciais, antes que se propusesse uma redação alternativa que representasse a dimensão de maneira mais clara e precisa. $O$ enunciado era: "Por favor indique seu nível de desacordo ou acordo com as seguintes afirmações a respeito de suas aulas de Educação Física."
A nova versão foi administrada a 60 alunos de secundária com idades entre 12 e 19. Os mesmos comentários sobre as instruções de elaboração e as formas assumidas pequenas alterações. Após a análise dos resultados psicométricos obtidos e uma revisão final pela equipe de investigação, chegou-se ao último instrumento adequado para a EF.

\section{Procedimento}

Após a autorização pelos órgãos diretivos das escolas para realizar a investigação, os alunos foram informados do propósito do estudo e os seus direitos como participantes na mesma. Os instrumentos para medir as diferentes variáveis foram administrados em sala de aula, sem a presença do(a) professor(a) da turma. Cada participante levou 10-20 minutos para completar os questionários. $\mathrm{O}$ anonimato dos respondentes foi garantido.

\section{Análise dos dados}

Foi realizada uma análise fatorial exploratória (EFA) dos dados coletados/obtidos para obter a estrutura de fator interno; a consistência interna foi analisada, a fim de determinar a fiabilidade (alfa) de Cronbach. O software de análises estatísticas SPSS $17,0 \AA$, foi utilizado como ferramenta de suporte. $E$ ainda, as análises foram desenvolvidas comparativamente entre meninos e meninas.

\section{Resultados}

\section{Análise de Itens e de homogeneidade da escala}

$\mathrm{Na}$ análise estatística dos itens foi mantida a distribuição de itens-fator observada no instrumento original (DUDA e NICHOLLS, 1992. BALAGUER et al, 1997).

Os critérios levados em conta para a manutenção de um item foram: coeficiente de correlação corrigido item-total $(C C / T-C) \geq 0,30$, desvio padrão $(S D)>1$, e todas as opções de resposta deveriam ter sido utilizadas em algum momento (NUNNALLY; BERNSTEIN, 1995). Os itens do primeiro fator (Satisfação/Diversão) apresentaram valores entre 3,85 para os itens $2 \mathrm{e}$ 7 (meninos) e 4,38 para o item 1 (meninos). Os $D P$ foram maiores do que 1 , variando entre 1,02 no item 8 nos meninos e 1,10 do item 7 nas meninas. A consistência interna desta dimensão foi adequada ( $\alpha 1=0,92$, meninos, $\alpha 2=0,95$ para 
meninas). Todos os CCIT-c apresentaram valores $>0,72$.

O segundo fator (Tédio) apresentou valores entre 1,87 para o item 3 e 2,25 no item 2 (ambos para meninos). O DP foi maior que 1 , oscilando entre 1,22 (item 2 meninas) e 1,40 (item 2 meninos). A consistência interna desta escala foi adequada ( $\alpha 1=0,85$, meninos, $\alpha 2=0,79$ para meninas).

Todos os CCIT-C apresentaram valores > 0,57 . Apesar destes dados e da consistência interna adequada, os resultados mostram que a exclusão do item 2 desta dimensão equivaleria a uma alpha 0,88 entre os homens, acima do 0,85 com os três itens, e se manteria um adequada 0,78 para meninas.
Em qualquer caso, estes dados devem ser considerados para análises posteriores, mas como a consistência interna obtida é adequada, o fator é mantido com os três itens (Tabela 1).

A correlação entre a pontuação de cada item e pontuação total em cada um dos componentes mostram correlações positivas com a dimensão teórica a qual pertencem e negativamente com o escore total da outra dimensão. Também neste caso destaca-se a atenção em relação ao item 2 em meninos, pois se pode observar como o valor negativo estava mais próximo de zero $(r=-.14)$; contudo, não mostra sobreposição com a dimensão teórica de Satisfação/Diversão. Decidiu-se, finalmente, manter o item, já que garante a homogeneidade em sua componente.

Tabela 1. Media $(M)$, desvio padrão $(D P)$, correlação item-total $(C C / T-c)$, alfa de cada escala teórica e a dimensão se algum ítem for eliminado ( $\alpha$ sem item), assimetria e curtosis $(n=224)$.

\begin{tabular}{|c|c|c|c|c|c|c|c|c|c|c|c|c|c|c|}
\hline \multirow[b]{2}{*}{ Escala: } & \multicolumn{7}{|c|}{ Meninos $(n=110)$} & \multicolumn{7}{|c|}{ Meninas $(n=114)$} \\
\hline & M & $D P$ & $C C / T-C$ & $C C$ & $\begin{array}{c}\alpha \text { sem } \\
\text { item }\end{array}$ & Assimetria & Curtosis & $M$ & $D P$ & $C C / T-C$ & $C C$ & $\begin{array}{c}\alpha \text { sem } \\
\text { item }\end{array}$ & Assimetria & Curtosis \\
\hline \multicolumn{15}{|l|}{$\begin{array}{l}\text { Satisfação/Diversão ( } \alpha_{1}=0,92, \text { meninos; } \alpha_{2} \\
\quad=0,95, \text { meninas) }\end{array}$} \\
\hline 1 Normalmente me divirto nas aulas de EF & 4.38 & 1.09 & .85 & -.36 & .89 & -1.35 & 1.03 & 4.02 & 1.17 & .92 & -.60 & .93 & -1.10 & .22 \\
\hline $\begin{array}{l}5 \text { Normalmente a EF me parece } \\
\text { interessante }\end{array}$ & 4.22 & 1.06 & .81 & -.35 & .90 & -1.48 & 1.64 & 3.85 & 1.19 & .87 & -.61 & .93 & -.95 & .13 \\
\hline 6 Quando faço EF parece que o tempo voa & 4.36 & 1.07 & .72 & -.42 & .92 & -1.64 & 1.14 & 3.94 & 1.28 & .90 & -.53 & .95 & -.93 & -.38 \\
\hline $\begin{array}{l}7 \text { Normalmente participo ativamente nas } \\
\text { aulas de EF }\end{array}$ & 4.29 & 1.08 & .79 & -.37 & .90 & -1.56 & 1.40 & 3.85 & 1.10 & .83 & -.46 & .94 & -.76 & -.28 \\
\hline 8 Normalmente passo bem fazendo EF & 4.33 & 1.02 & .82 & -.33 & .90 & -.18 & 1.00 & 3.98 & 1.18 & .88 & -.62 & .93 & -1.01 & .02 \\
\hline \multicolumn{15}{|l|}{$\begin{array}{l}\text { Tédio }\left(\alpha_{1}=0,85, \text { meninos; } \alpha_{2}=0,79,\right. \\
\text { meninas) }\end{array}$} \\
\hline $\begin{array}{l}2 \text { Nas aulas de EF, quase sempre sonho } \\
\text { acordado em vez de pensar no que } \\
\text { estou realmente fazendo }\end{array}$ & 2.25 & 1.40 & .61 & -.14 & .88 & .81 & -.66 & 2.06 & 1.22 & .57 & -.43 & .78 & .92 & -.21 \\
\hline $\begin{array}{l}3 \text { Nas aulas de EF, normalmente me entedio } \\
\text { (aborreço) }\end{array}$ & 1.87 & 1.31 & .75 & -.47 & .75 & 1.35 & .47 & 2.05 & 1.26 & .58 & -.55 & .77 & 1.03 & -.04 \\
\hline $\begin{array}{l}4 \mathrm{Na} E F \text { desejo que a aula termine } \\
\text { rapidamente }\end{array}$ & 2.03 & 1.34 & .78 & -.49 & .71 & 1.00 & -.36 & 2.15 & 1.34 & .75 & -.57 & .58 & .92 & -.44 \\
\hline
\end{tabular}

\section{Análise da estrutura interna}

Tal como recomendado por Bollen (1994), os índices de assimetria e curtose estão perto de $0 \mathrm{e}$ $<2$. Foi realizado um EFA usando o método de extração de componentes principais (PCA), exigindo-se um mínimo de correlação 0,40 para que cada item fosse importante dentro do fator, como observado por Stevens (1992). A medida de adequação da amostra Kaiser-Meyer-Olkin (KMO $=0,84$, meninos, $\mathrm{KMO}=0,88$, meninas) é aceitável e o teste de esfericidade de Bartlett foi estatisticamente significativo $\left(\chi^{2}{ }_{(28)}=617,361, p<\right.$ 0.000 em meninos e, $\chi_{(28)}^{2}=766,307, p<0,000$ em meninas), permitindo a conclusão da pertinência da aplicação do EFA. Os resultados confirmam que a extração de dois fatores exige três interações: Satisfação/Diversão e Tédio (Tabela 2).

A análise de correlação indica que as duas dimensões são significativamente correlacionados negativamente entre si $(r=-0,42$, meninos; $r=$ $0,62$, meninas; $p<0.01)$. Com uma porcentagem total de variância explicada de $88,2 \%$ (84,3\% para os meninos e $88,1 \%$ em meninas), os resultados da análise exploratória de fatores expõem a estrutura dimensional da escala em dois fatores com saturação dos itens acima de 0,70 . Portanto, - SSI adaptado para a EF apresenta-se com duas dimensões (8 itens) que se referem à Satisfação/Diversão (itens $1,8,5,7,6$ ) e Tédio (itens 2, 4, 3) (Tabela 2 .) 
Tabela 2. Estrutura fatorial rotada, comunicações, autovalores, alpha de Cronbach e porcentagem de variação explicada ( $\mathrm{N}=224)$. Método de extração: Análise de componentes principais. Coeficiente de visualização 0,40 .

\begin{tabular}{|c|c|c|c|c|c|c|}
\hline \multirow[b]{2}{*}{ Fator } & \multicolumn{3}{|c|}{ Meninos } & \multicolumn{3}{|c|}{ Meninas } \\
\hline & $\mathrm{F} 1$ & $\mathrm{~F} 2$ & $h^{2}$ & $\mathrm{~F} 1$ & $\mathrm{~F} 2$ & $h^{2}$ \\
\hline 1 Normalmente me divirto nas aulas de EF & .90 & & .83 & .88 & & .90 \\
\hline 8 Normalmente me lo paso bien haciendo Educación Física & .89 & & .78 & .86 & & .87 \\
\hline 5 Normalmente a EF me parece interessante & .87 & & .66 & .86 & & .85 \\
\hline 7 Normalmente participo ativamente nas aulas de EF & .85 & & .76 & .89 & & .82 \\
\hline 6 Quando faço EF parece que o tempo voa & .76 & & .81 & .83 & & .75 \\
\hline $\begin{array}{l}2 \text { Nas aulas de EF, quase sempre sonho acordado em vez de pensar } \\
\text { no que estou realmente fazendo }\end{array}$ & & .87 & .77 & & .82 & .70 \\
\hline $4 \mathrm{Na}$ EF desejo que a aula termine rapidamente & & .84 & .80 & & .84 & .82 \\
\hline 3 Nas aulas de EF, normalmente me entedio (aborreço) & & .83 & .83 & & .70 & .62 \\
\hline Autovalor & 4.58 & 1.64 & & 5.25 & 1.07 & \\
\hline Porcentagem de variação explicada & 57.31 & 20.51 & 77.83 & 65.68 & 13.42 & \\
\hline Kaiser-Meyer-Olkin (KMO) & \multicolumn{3}{|c|}{.84} & \multicolumn{3}{|c|}{.88} \\
\hline Esfericidade de Bartlett & \multicolumn{3}{|c|}{$\chi_{(28)}^{2}=617,361 ; p<.000$} & \multicolumn{3}{|c|}{$\chi_{(28)}^{2}=766,307 ; p<.000$} \\
\hline Alplha de Cronbach & .92 & .85 & & $.95^{n}$ & .79 & \\
\hline
\end{tabular}

Nota. F1 = Satisfação/Diversão; F2 = Tédio

\section{Conclusões}

Um dos aspectos interessantes desta escala é que foi elaborada especificamente para estudantes de educação secundária ${ }^{3}$ (entre 12 e 19 anos), com uma amostra muito semelhante para ambos os sexos. Além disso, este estudo desenvolveu-se com uma amostra aceitável para apresentar os diversos resultados obtidos com suficientes garantias psicométricas em relação à escala SSI adaptada à EF. Isto significa que o tamanho da amostra foi adequado porque, como estimado por Tabachnick e Fidell (1989), entre 100 e 200 casos são suficientes para realizar análises fatoriais.

Estes resultados sugerem que a SSI adaptada para a EF tem propriedades psicométricas (altas fiabilidade e validade de construto) bastante aceitáveis e está conforme com trabalhos já encontrados em ambas as escalas (DUDA; NICHOLLS, 1992; BALAGUER et al, 1997, e outros).

Como evidência de validade baseada na estrutura interna (validade fatorial), a análise fatorial exploratória revelou a estrutura bidimensional da escala em dois fatores completamente independentes, como na obra de Balaguer et al. (1997) em SSI, e na de Castillo, et al. (2001), em CSI, na direção sugerida pelos autores originais (NICHOLLS et al, 1985, 1989. DUDA; NICHOLLS, 1992). Vale ressaltar que o percentual total da variância explicada é de $88,2 \%$, com saturação dos itens acima de 0,70 .
Em relação à fiabilidade desses dois fatores, ambos apresentam consistência interna adequada (embora menor que a obtida por Balaguer et al. [1997], nos esportes) e todos os itens se relacionam de maneira significativa com os fatores aos quais pertencem, exibindo uma alta capacidade discriminativa. Isso mostra que o pertencimento de cada item a seu fator mantém uma visão ampla das diversas manifestações do construto por não ter que remover qualquer um deles para que a escalar funcione corretamente. No entanto, se pode comprovar, como no trabalho de Balaguer et al. (1997) e Castillo et al. (2001), que a remoção do item 2 do fator Tédio promoveria um alfa maior do que com ele. No entanto, foi decidido mantê-lo porque a consistência interna com o mesmo é apropriada e se pretende levar isto em conta para análises posteriores e com amostras diferentes.

Os resultados do CCIT-C apresentam valores muito aceitáveis, sendo na dimensão Satisfação/Diversão acima de 0,72 em meninos e acima de 0,83 para as meninas.

No caso de Tédio, os valores são mais discretos, embora também positivos, acima de 0,61 nos meninos e 0,57 nas meninas.

Em geral, podemos dizer que todos os itens têm um poder discriminativo aceitável e, juntos, ambos medem com precisão a Satisfação/Diversão ou o Tédio.

\footnotetext{
${ }^{3}$ Equivalência com níveis de ensino brasileiros já apontada em nota anterior.
} 
Concluindo, deve-se destacar que as propriedades da versão adaptada do SSI para a EF são semelhantes às do teste original e os resultados obtidos mostram que a escala tem propriedades psicométricas suficientemente robustas para ser utilizada como uma ferramenta para avaliar Satisfação na EF. É importante ressaltar, no entanto, que os resultados deste estudo devem ser interpretados à luz da amostra utilizada, com foco em estudantes de secundária. É, portanto, clara a necessidade de replicar este estudo em uma população mais heterogênea, o que permite avaliar se a estrutura bi fatorial encontrada nesta pesquisa aparece também em outras amostras, com características diferentes. Na mesma linha, é necessário complementar os estudos com análise fatorial confirmatória que apoiem com precisão a composição fatorial de construto e o ajuste dos dados a este.

\section{Referências}

BALAGUER, I.; ATIENZA, F. L.; CASTILLO, I.; MORENO, Y.; DUDA, J. L. Factorial structure of measures of satisfaction/interest in sport and classroom in the case of Spanish adolescents. Abstracts of 4th. European Conference of Psychological Assessment, 1997, Lisbon: Portugal, 1997, p.76.

BALAGUER, I. Un studio sobre los predictors de los estilos de vida saludables de los adolescents valencianos. Direcció General de Salut Pública (I.V.E.S.P., 011/1999). Valencia: Consellería de Sanitat de la Generalitat Valenciana, 2000.

BOLLEN, K.; LONG, J. S. Testing structural equation models. Newbury Park, CA: Sage, 1994.

CALABUIG, F.; CRESPO, J. Uso del método Delphi para la elaboración de una medida de la calidad percibida de los espectadores de eventos deportivos. Retos: Nuevas tendencias en Educación Física, Deporte y Recreación, Murcia, v.16, p.21-25, 2009.

CASTILLO, I.; BALAGUER, I.; DUDA, J. L. Perspectivas de meta de los adolescentes en el contexto académico. Psicothema, Oviedo, v.13, n.1, p.79-86, 2001.

CASTILLO, I. BALAGUER, I. DUDA, J. L. Las perspectivas de meta de los adolescentes en el contexto deportivo. Psicothema, Oviedo, v.14, n.2, p.280-287, 2002.

CASTILLO, I.; BALAGUER, I.; DUDA, J. L.; GARCÍA-MERITA, M. L. Factores psicosociales asociados con la participación deportiva en la adolescencia. Revista Latinoamericana de Psicología, Bogotá, v.36, n.3, p.505-515, 2004.

DUDA, J. L. Goals: a social-cognitive approach to the study of achievement motivation in sport. In: SINGER, R. N. MURPHEY, M. TENNANT, L. (Eds.). Handbook of research in Sport Psychology. New York: Macmillan, p. 421-436, 1993.

DUDA, J. L.; NICHOLLS, J. G. Dimensions of achievement motivation in schoolwork and sport. Journal of Educational Psychology, Washington, v.84, n.3, p.290-299, 1992.

NICHOLLS, J. G.; CHEUNG, P. C.; LAUER, J.; PATASHNICK, M. Individual differences in academic motivation: Perceived ability, goals, beliefs, and values. Learning and Individual Differences, New Haven, v.1, p.63-84, 1989.

NICHOLLS, J. G.; PATASHNICK, M.; NOLEN, S. $B$. Adolescents' theories of education. Journal of Educational Psychology, Washington, v. 77, p.683-692, 1985.

NUNNALLY, J. C.; BERNSTEIN, I. (1995). Teoría psicométrica. Madrid: McGraw-Hill.

NUVIALA, A.; TAMAYO, J. A.; IRANZO, J.; FALCÓN, D. Creación, diseño, validación y puesta en práctica de un instrumento de medición de la satisfacción de usuarios de organizaciones que prestan servicios deportivos. Retos: Nuevas tendencias en Educación Física, Deporte y Recreación, Murcia, v. 14, p.10-16, 2008.

OSTERLIND, S. J. Constructing Test Items. Londres: Kluwer Academic Publishers. 1989.

ROBERTS, G. C. Children's achievement motivation in sport. In: NICHOLLS, J. G. (Ed.). Advances in motivation and achievement: The development of achievement motivation (vol. 3,). Greenwich, Conn.: JAI Press. 1984, p. 251-281.

SPAAN, M. Test and item specifications development. Language Assessment Quarterly, Philadelphia, v.3, p.71-79, 2006.

STEVENS, J. Applied multivariate statistics for the social sciences. Hillsdale, NJ: Lawrence Erlbaum. 1992.

STUCKY-ROPP, R. C.; DILORENZO, T. M. Determinants of exercise in children. Preventive Medicine, Montreal, v. 22, p.880-889, 1993.

TABACHNICK, B. G.; FIDELL, L. S. Using multivariate statistics. Nueva York: Harper Collins. 1989. 


\section{Endereço:}

Antonio Baena-Extremera

Departamento Actividad Física y Deporte

Facultad de Ciencias del Deporte. Universidad de

Murcia

C/ Argentina, s/n

Santiago de la Ribera Murcia España

30720

e-mail: abaenaextrem@um.es

Recebido em: 19 de junho de 2012.

Aceito em: 12 de dezembro de 2012.

\section{ANEXO}

Instrumento de Coleta de dados (SSI) Sport Satisfaction Instrument.

Indica tu grado de acuerdo o desacuerdo, siendo muy en desacuerdo (1) a muy de acuerdo (5).

1. Normalmente me divierto en las clases de Educación Física

2. En las clases de Educación Física a menudo sueño despierto en vez de pensar en lo que hago realmente

3. En las clases de Educación Física, normalmente me aburro

4. En Educación Física deseo que la clase termine rápidamente

5. Normalmente encuentro la Educación Física interesante

6. Cuando hago Educación Física parece que el tiempo vuela

7. Normalmente participo activamente en las clases de Educación Física

8 Normalmente me lo paso bien haciendo Educación Física

Fonte: (DUDA e NICHOLLS, 1992), adaptado para o espanhol por BALAGUER et al. (1997). 